\title{
Size of the coming solar cycle 24 based on Ohl's Precursor Method, final estimate
}

\author{
R. P. Kane \\ Instituto Nacional de Pesquisas Espacias, INPE C.P. 515, 12201-970 São Jose dos Campos, SP, Brazil \\ Received: 5 January 2010 - Revised: 13 July 2010 - Accepted: 14 July 2010 - Published: 16 July 2010
}

\begin{abstract}
In Ohl's Precursor Method (Ohl, 1966, 1976), the geomagnetic activity during the declining phase of a sunspot cycle is shown to be well correlated with the size (maximum sunspot number $R z(\max )$ ) of the next cycle. For solar cycle 24 , Kane (2007a) used $a a(\min )=15.5$ (12-month running mean), which occurred during March-May of 2006 and made a preliminary estimate $R z(\max )=124 \pm 26$ (12-month running mean). However, in the next few months, the $a a$ index first increased and then decreased to a new low value of 14.8 in July 2007 . With this new low value, the prediction was $R z(\max )=117 \pm 26$ (12-month running mean). However, even this proved a false signal. Since then, the aa values have decreased considerably and the last 12 -monthly value is 8.7 , centered at May 2009. For solar cycle 24, using $a a(\min )=8.7$, the latest prediction is, $R z(\max )=58.0 \pm 25.0$.
\end{abstract}

\section{Introduction}

Prediction of the magnitude of the sunspot maximum $R z(\max )$ is important for planning satellite launching For cycle 23, NOAA's Space Environment Center (SEC) recruited a scientific panel to assess the likely development of cycle 23 and their report entitled "Solar Cycle 23 Project: Summary and Panel Findings," later published as Joselyn et al. (1997), mentioned (i) a range of 160-200 of $R z(\max )$ of cycle 23 as obtained by considering the even/odd behavior and (ii) a range $110-160$ of $R z(\max )$ by other methods. The panel gave the greatest weight to precursor methods, since they have proved to be the most successful technique for solar activity predictions in the past. The precursor methods invoke a so- lar dynamo concept, whereby the polar field in the declining phase and at minimum is the seed of future toroidal fields within the Sun that will cause solar activity (e.g., Schatten and Pesnell, 1993). In one of the precursor methods (Ohl's method), geomagnetic $a a$ indices at solar minimum are seen to be well correlated with the succeeding $R z(\max ) \quad$ (Ohl, 1966, 1976; Brown and Williams, 1969; Ohl and Ohl, 1979; Sargent, 1978). Since then, Kane (1978, 1987, 1992, 1997, 1998), Wilson (1988a, b, 1992), and Wilson et al. (1998) have been studying this aspect for the past three decades. For cycle 24, Kane (2007a) evaluated $R z(\max )$ as $124 \pm 26$ using $a a(\min )=15.5$ which seemed to be a minimum at that time. However, some workers (notably L. Svalgaard, private communication) were apprehensive that since $a a(\mathrm{~min})$ usually occurs a few months later than $R z(\min )$, and $R z(\min )$ had not yet occurred in 2006, the $a a(\min )=15.5$ in March 2006 could be a false alarm. A Solar Cycle 24 Prediction Panel composed of international scientists and presided by D. Biesecker, (details given in http://www.sec.noaa.gov/ SolarCycle/SC24/index.html), issued on 25 April 2007, a consensus opinion that cycle 24 would commence in March 2008 ( \pm 6 months) and two consensus opinions, that the solar maximum would be $140 \pm 20$ in October 2011 or $90 \pm 10$ in August 2012. As it happened, the sunspot minimum was nowhere near the range March 2008 ( \pm 6 months) mentioned by them, and the old cycle 23 seems to have ended and the new cycle 24 commenced only in the end of 2009. The sunspot numbers seem to have gone through a minimum of zero in August 2009. In this note, the situation of $R z(\max )$ vis-a-vis $a a(\min )$ is reexamined to see whether a final estimate can be given for $R z(\max )$ of cycle 24 .

\section{Correspondence to: R. P. Kane}

(kane@dge.inpe.br)

Published by Copernicus Publications on behalf of the European Geosciences Union. 


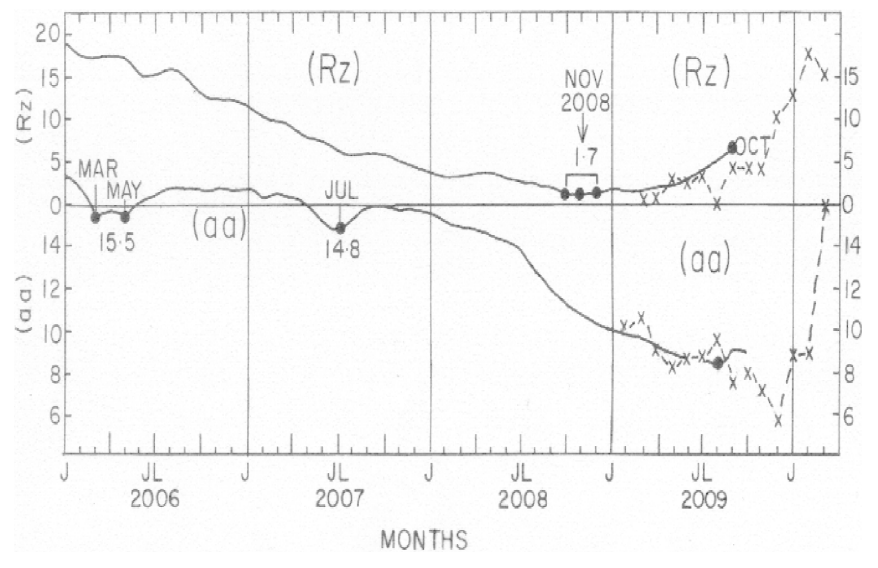

Fig. 1. Plot of the 12-month running means of sunspot number $R z$ and geomagnetic index $a a$ for January 2006 onwards. Vertical lines mark the month January. Minima are shown by big dots and the corresponding months are indicated.

\section{Data}

The data used are the geomagnetic $a a$ indices (the antipodal amplitudes deduced from the $\mathrm{K}$ index of Greenwich, England, and Melbourne, Australia; Mayaud, 1973) available at NOAA ftp://ftp.ngdc.noaa.gov/STP/SOLAR DATA/RELATED_INDICES/AA_INDEX/ and international sunspot numbers $R z$ (McKinnon, 1987, and further data from NOAA websites ftp and ftp://ftp.ngdc.noaa.gov/STP/ SOLAR_DATA/SUNSPOT_NUMBERS). For $a a$ indices, data are available from 1868 onward only (cycle 11). However, for 1844-1880, Nevanlinna and Kataja (1993) generated an "equivalent" $a a$ index based on the declination data from Helsinki and, for the overlapping period of 13 years 1868-1880, they found a very high correlation (0.99). Hence, we have now aa index data for cycle 9 and 10 also (annual values) and for cycle 11 onward (monthly values).

\section{Present situation}

The origin and development of sunspot numbers are described recently in Clette et al. (2007) and Vaquero (2007). The monthly values of $R z$ and $a a$ indices vary erratically from month to month. Hence, 12-month running means were evaluated and used. (This is simple average of 12 consecutive monthly values. The centering is half-a-month displaced. To get the centering correct, some workers use 13 consecutive monthly values, with half weight for the first and last values. However, the difference between the two estimates is negligibly small). As shown in Kane (2007a), for cycles 12-23, the $R z(\min )$ occurred earlier than $a a(\min )$ by $3,5,-2,2,14$, $8,16,12,7,7,4,16,9$ months. Thus, except for cycle 14 where $R z(\max )$ occurred later by 2 months ( -2 italicized), the $a a(\mathrm{~min})$ have occurred with delays ranging from 2 to 16 months. Figure 1 shows the plots of the 12-month running means of $R z$ and $a a$ indices for January 2006 onwards. The following may be noted:

1. The 12-month running mean $R z(\min )$ seems to have occurred only recently. The mean 1.7 occurred in three successive months October, November, December 2008, followed by values 1.9, 1.9, 2.2, 2.3, 2.3, 3.1, 4.1, 5.5, 6.7 till September 2009. Further, the actual monthly values for July 2009-March indicate that the sunspot activity has started rising. For January-March 2010 , monthly values were $13.1,18.6,15.4$

2. The 12-month running mean $a a(\min )$ seemed to have occurred first centered at March 2006 and May 2006 with a value 15.5. In Kane (2007a), this value was used in the regression equation $\quad R z(\max )=(-24.9 \pm 18.1)+(9.6 \pm 0.1 .2) a a(\min )$ and yielded the preliminary estimate prediction $R z(\max )=124 \pm 26$.

3. However, in retrospect, this seems to have been a false alarm. The $a a$ values increased in the next few months but decreased later considerably, and attained a second minimum centered at July $2007, a a(\mathrm{~min})=14.8$, smaller in magnitude than the first minimum and 15 months later. This also has proved a false signal and after rising for the next six months, the aa values have decreased considerably. The last 12 -monthly running mean for aa index is 8.7, centered on June 2009. If this value 8.7 is used as aa $(\mathrm{min})$, the prediction becomes $R z(\max )=58.0 \pm 25.0$.

\section{Conclusion and discussion}

In Ohl's Precursor Method, the geomagnetic activity during the declining phase of a sunspot cycle is shown to be well correlated with the size (maximum sunspot number $R z(\max )$ of the next cycle. Kane (1997, 1998, and references therein) and Wilson, Hathaway and Reichmann (1998 and references therein) have been using the $a a$ index during the sunspot minimum year as representative of the geomagnetic activity. For solar cycle 24 , using $a a(\mathrm{~min})=8.7$, the latest prediction is $R z(\max )=58.0 \pm 25.0$.

Recently, Du et al. (2008, 2009) examined the Ohl method more critically, and pointed out that a higher correlation does not necessarily mean a successful prediction, In their analyses, a higher correlation often yielded a failure prediction. Therefore, they suggest that when a prediction is obtained, its rationality should be analyzed. As one test, they suggest that the prediction could be considered reliable if the prediction lies very near the regression line and the correlation increases. We tried this criterion. For cycles 9-23, the correlation was +0.918 . When the value 8.7 for $a a(\mathrm{~min})$ in May 2009 was used with predicted $R z(\max )$ as 58 , the correlation was slightly higher $(+0.931)$. Thus the predicted value 
$R z(\max )=58 \pm 25$ for cycle 24 should be fairly reliable, and it is in the low $R z(\max )$ category.

For cycle 24 , there are predictions in a very wide range (see list and references in Kane, 2007a), namely, (a) <70 (three predictions), (b) 70-90 (eight predictions), (c) 90-110 (eight predictions), (d) 110-130 (ten predictions), (e) 130150 (seven predictions), (f) 150-170 (three predictions), and $(\mathrm{g})>170$ (four predictions). Our latest prediction of $R z(\max )=58 \pm 25$ is in the (a) range of $<70$, much lower than the average of all predictions $(\sim 115)$. Thus, if our prediction comes true, predictions in the high range $(\mathrm{g})$ like $R z(\max )$ 150-180 (Dikpati et al., 2006), $R z(\max )=180$ (Tsirulnik et al., 1997), $R z(\max )=185$ (Horstman, 2005) would prove grossly erroneous, while predictions in the very low range (a) $R z(\max )=42 \pm 34$ (Clilverd et al., 2006) and $R z(\max )<50$ (Badalyan et al., 2001) would prove to be true.

Incidentally, the present author published predictions for cycle 24 by using other methods also and got the results as (i) Kane (2007b) based on solar activity at different solar latitudes, $R z(\max )=130$, (ii) Kane $(2007 \mathrm{c})$ based on extrapolation of spectral components, $R z(\max )=92$, (iii) Kane (2008a) based on solar cycle lengths, $R z(\max )=94$, and (iv) Kane (2008b) based on the Ohl-Kopecky rule and the three-cycle periodicity scheme, $R z(\max )=106$. Almost all these (except 130 in Kane, 2007b) are below the statistical average of $\sim 115$ for the 23 cycles. Thus, cycle 24 is likely to be below average. Recently, a review on this solar minimum has been published by Russell et al. (2010).

Acknowledgements. This work was partially supported by FNDCT, Brazil, under Contract No. FINEP-537/CT.

Topical Editor R. Forsyth thanks Z. Du and J. Vaquero for their help in evaluating this paper.

\section{References}

Badalyan, O. G., Obridko, V., and Sykora, N. J.: Brightness of the coronal green line and prediction for activity cycles 23 and 24, Solar Phys., 199, 421-435, 2001.

Brown, G. M. and Williams, W. R.: Some properties of the day-today variability of $\mathrm{Sq}(\mathrm{H})$, Planet. Space Sci., 17, 455-470, 1969.

Clette, F., Berghmans, D., Vanlommel, P., van der Linden, R. A. M., Koeckelenbergh, A., and Wauters, L.: From the Wolf number to the International Sunspot Index: 25 years of SIDC, Adv. Space Res., 40, 919-928, 2007.

Clilverd, M. A., Clarke, E., Ulich, T., Rishbeth, H., and Jarvis, M. J.: Predicting Solar Cycle 24 and beyond, Space Weather, 4, S09005, doi:10.1029/2005SW000207, 2006.

Dikpati, M., de Toma, G., and Gilman, P. A.: Predicting the strength of solar cycle 24 using a fluxtransport dynamo-based tool, Geophys. Res. Lett., 33, L05102, doi:10.1029/2005GL025221, 2006.

Du, Zhan-Le, Wang, Hua-Ning, and Zhang, Li-Yun:, A Running Average Method for Predicting the Size and Length of a Solar Cycle, Chin. J. Astron. Astrophys., 8 , 477-488, 2008.

Du, Z. L., Li, R., and Wang, H. N.: The predictive power of Ohl's precursor method, Astrophys. J., 138, 1998-2001, 2009.
Horstman, M.: Varying solar flux models and their effect on the future debris environment Projection, The Orbital Debris Quarterly News, 9 (January 2005), 4-5, 2005.

Joselyn, J. A., Anderson, J. B., Coffey, H., Harvey, K., Hathaway, D., Heckman, G., Hildner, E., Mende, W., Schatten, K., Thompson, R., Thomson, A. W. P., and White, O. R.: Panel achieves consensus prediction of solar cycle 23, Eos. Trans. AGU, 78, 205, 1997.

Kane, R. P.: Predicted intensity of the solar maximum, Nature, 274, 139-140, 1978.

Kane, R. P.: Prediction of the maximum annual mean sunspot number in the coming solar maximum epoch, Solar Phys., 108, 415416, 1987.

Kane, R. P.: Did predictions of the maximum sunspot number for solar cycle no. 22 come true?, Solar Phys., 140, 171-180, 1992.

Kane, R. P.: A preliminary estimate of the size of the coming solar cycle 23, based on the Ohl's precursor method, Geophys. Res. Lett., 24, 1899-1902, 1997.

Kane, R. P.: Correction to 'A preliminary estimate of the size of the coming solar cycle 23, based on Ohl's precursor method' by R. P. Kane, Geophys. Res. Lett., 25, 3121, 1998.

Kane, R. P.: A Preliminary Estimate of the Size of the Coming Solar Cycle 24, based on Ohl's Precursor Method, Solar Phys., 243, 205-217, 2007a.

Kane, R. P.: Solar cycle predictions based on solar activity at different solar latitudes, Solar Phys., 246(2), 471-485, $2007 \mathrm{~b}$.

Kane, R. P.: Solar Cycle Predictions Based on Extrapolation of Spectral Components: An Update, Solar Phys., 246, 487-493, 2007c.

Kane, R. P.: Prediction of Solar Cycle Maximum Using Solar Cycle Lengths, Solar Phys., 248, 203-209, 2008a.

Kane, R. P.: Prediction of solar cycle 24 based on the GnevyshevOhl-Kopecky rule and the three-cycle periodicity scheme, Ann. Geophys., 26, 3329-3339, doi:10.5194/angeo-26-3329-2008, 2008 b.

Mayaud, P. N.: A hundred year series of geomagnetic data, 18681967, indices aa, in: IAGA Bull., 33, IUGG Publication Office, Paris, pp. 262, 1973.

McKinnon, J. A.: Sunspot numbers 1610-1985, in: UAG Report 95, NOAA, Boulder, pp. 112, 1987.

Nevanlinna, H. and Kataja, E.: An extension of geomagnetic activity series aa for two solar cycles (1844-1868), Geophys. Res. Lett., 20, 2703-2806, 1993.

Ohl, A. I.: Wolf s Number Prediction for the Maximum of the Cycle 20, Solnice Dani, 12, 84, 1966.

Ohl, A. I.: A preliminary forecast of some parameters of cycle No. 21 of the solar activity, Solnice Danie, 9, 73-75, 1976.

Ohl, A. I. and Ohl, G. I.: A new method of very long-term prediction of solar activity, in: Solar-Terrestrial Predictions Proceedings, edited by: Donnely, R. F., NOAA/Space Environmental Laboratories, Boulder, pp. 258, 1979.

Russell, C. T., Luhmann, J. G., and Jian, L. K.: How unprecedented a solar minimum?, Rev. Geophys., 48, RG2004, doi:10.1029/2009RG000316, 2010.

Sargent III, H. H.: A prediction for the next sunspot cycle, in: Proc. 28th IEEE Vehicular Technology Conf. Denver, Colo. (1978), 490-496, 1978.

Schatten, K. H. and Pesnell, W. D.: An Early Solar Dynamo Prediction: Cycle $23 \sim$ Cycle 22, Geophys. Res. Lett., 20, 2275-2278, 
1993.

Tsirulnik, L. B., Kuznetsova, T. V., and Oraevsky, V. N.: Forecasting the 23rd and 24th solar cycles on the basis of MGM spectrum, Adv. Space Res., 20, 2369-2372, 1997.

Vaquero, J. M.: Historical Sunspot Observations: A Review, Adv. Space Res., 40, 929-941, 2007.

Wilson, R. M.: A prediction for the size of sunspot cycle 22, Geophys. Res. Lett., 15, 125-128, 1988a.
Wilson, R. M.: Bimodality and the Hale cycle, Solar Phys., 117, 269-278, 1988b.

Wilson, R. M.: An early estimate for the size of cycle 23, Solar Phys., 140, 181-193, 1992.

Wilson, R. M., Hathaway, D. H., and Reichmann, E. J.: An estimate for the size of cycle 23 based on near minimum conditionsd, J. Geophys. Res., 103, 6595-6603, 1998. 